\title{
Spectroscopic and photometric properties of red stars in the bar of the Large Magellanic Cloud
}

\author{
Tom Lloyd Evans \\ School of Physics and Astronomy, University of St Andrews, North \\ Haugh, St Andrews, Fife, Scotland KY16 9SS
}

\begin{abstract}
Spectroscopy and variability studies of red stars in the Bar of the LMC enable the stars to be classed as spectral type M, S or C and as variables of class L, SR or Mira. The results are compared with data for Magellanic Cloud star clusters and for the galactic field. The number of $S$ stars relative to carbon stars is generally high in comparison to theoretical expectation.
\end{abstract}

\section{Introduction}

Lloyd Evans (1972) surveyed a field in the Bar of the LMC, centred on the variable star S Dor, with the 1.9-m reflector of the Radcliffe Observatory, Pretoria. Some results for Mira variables have been given by Glass \& Lloyd Evans (1981, 2003) and for semiregular variables by Lloyd Evans (1992). This paper gives spectroscopic results for a large sample.

\section{Variability and period determination}

Pairs of $V$ and $I$ plates were used to identify red stars and so to exclude short period blue variables such as Cepheids, so that periods could be determined from plates which were only taken near New Moon. A total of $44 \mathrm{~V}$ plates were taken in 1965-71 (Lloyd Evans 1972). Periods have been determined, from the maxima and minima in their light curves, for 83 stars: spectra were obtained for all but eight of them, while spectra are available for eight stars of smaller amplitude for which periods were not found.

Several Miras which were not included in the initial sample of Glass \& Lloyd Evans (1981) were found, but most of the stars are regarded as semiregular.

\section{Spectroscopy}

Spectroscopic observations were made using the Unit Spectrograph and RPCS detector on the $1.9-\mathrm{m}$ telescope at Sutherland. The $300 \mathrm{gmm}^{-1}$ grating was used to give a resolution of $0.7 \mathrm{~nm}$ over the range $430-680 \mathrm{~nm}$ in $1983 \mathrm{March}$ and the more efficient 400 grooves $\mathrm{mm}^{-1}$ grating was used to give a resolution of $0.5 \mathrm{~nm}$ and range $520-770 \mathrm{~nm}$ in 1984-86. The spectra were classified using a set of spectra of galactic $\mathrm{M}, \mathrm{S}$ and $\mathrm{C}$ stars. 


\section{Selection effects on the spectral type distribution}

Stars were selected in different ways in the various fields and this needs to be taken into account in the analysis of the spectral type distributions in these samples. Stars in the Magellanic Cloud clusters were selected as red in $V-I$ in early work (Lloyd Evans 1980) but the later and more complete studies (Bessell, Wood \& Lloyd Evans 1983; Lloyd Evans 1983, 1984) were based on $J H K$ photometry. The data for $\mathrm{S}$ and carbon stars are fairly complete, but no attempt was made to extend the spectroscopic investigation down the giant branch to obtain data for $\mathrm{M}$ stars below the $\mathrm{M}-\mathrm{S}$ transition.

Semiregular (SR) and irregular (L) variables have been combined in group $\mathrm{SR}$, as the distinction is often lost because of inadequate observations. The comparison of numbers of $\mathrm{M}, \mathrm{S}$ and $\mathrm{C}$ stars requires that all the types are combined in the final samples, as carbon stars have systematically smaller amplitudes in visible light and are often classified as SR rather than Mira when an amplitude criterion derived from $M$ stars is used to distinguish the different types of variable.

The data for the Magellanic Cloud samples depend essentially on a single, fairly complete survey of each field or cluster, whereas those for the Galaxy have been assembled from many different studies using a variety of techniques to select the stars. Detection of the more marginal examples of the S stars, especially, require spectra of good quality. Comparison of the figures for the first named variables in each constellation, represented by variables $\mathrm{R}-\mathrm{ZZ}$ in the more populous constellations, with the whole sample provided by the 1985 edition of the GCVS shows that the ratios of $n(S+C) / n(M)$ and $n(S) / n(C)$ are each increased by about $30 \%$ in a sample approximately five times smaller while the proportion of carbon stars is almost unchanged (Table 1).

The $\mathrm{M}$ and $\mathrm{S}$ stars in the Magellanic Cloud clusters are unlikely to be significantly variable. There are no carbon Miras and only one known semiregular in the cluster sample. Three heavily-obscured carbon Miras of very long period (Nishida et al, 2000) and two stars detected in the mid-infrared (Tanabe et al. 1999) have been excluded as they are very faint in visible light and their counterparts will generally not have been found in the fields.

SC and CS stars, which are likely to be massive AGB stars in the Hot Bottom Burning (HBB) phase of evolution, have been omitted. Only one star in the Large Magellanic Cloud sample, the 540-d period CS star C2 (Glass \& Lloyd Evans 2003), is thought to be of this type. The ${ }^{13} \mathrm{C}$-rich $\mathbf{J}$ stars have not been identified consistently in the complete sample and have been retained by default, though they most probably do not participate in the $\mathrm{M}-\mathrm{S}-\mathrm{C}$ sequence. They amount to about $15 \%$ of the cool carbon stars.

\section{Results}

\subsection{The spectral types}

The $M$ types are systematically earlier than those typical of red variables in the Galaxy. The average for the M stars is close to M4 and none is later than M7. Most of the S stars show relatively weak enhancements of $\mathrm{ZrO}$ and only one is a nearly-pure $\mathrm{S}$ star of type S6/6. The carbon stars in the Magellanic 
Clouds generally have quite weak $\mathrm{NaD}$, which is easily overwhelmed by night sky emission at this low resolution (Lloyd Evans 1980), so only the second parameter of the $\mathrm{C}$ classification system, the $\mathrm{C}_{2}$ bandstrength, is available. The well-known difference in the ratio of carbon stars to $M$ stars is apparent in comparing the LMC Bar field with the solar neighbourhood.

\subsection{The S:C number ratio}

There is a difference between the situation in the two types of sample, the star clusters and the field. Few of the cluster stars are variables and in this case the true $\mathrm{M}-\mathrm{S}-\mathrm{C}$ transition is seen as stars of similar composition and a small range of age and hence evolutionary stage ascend the giant branch. The field sample comprises variable stars which, although in different stages of evolution of their atmospheric compositions, are at the same stage, later than in the clusters, of evolution of their envelopes towards increasing variability and ultimate detachment from the star. These stars may represent a wide range of age, mass and initial metal content, in both the Galaxy and the Bar of the LMC. The variable $S$ stars in the field are probably more massive or more metal-rich than those in the clusters. They are undergoing the $\mathrm{M}$ to $\mathrm{C}$ transition at higher luminosity on the giant branch; this is supported by their spectral types, some of which are later than those of any of the S stars in the clusters.

Table 1. Numbers of stars of types M, S and C

\begin{tabular}{lrrrccc}
\hline Sample & $\mathrm{n}(\mathrm{M})$ & $\mathrm{n}(\mathrm{S})$ & $\mathrm{n}(\mathrm{C})$ & $(\mathrm{S}+\mathrm{C}) / \mathrm{M}$ & $\mathrm{C} / \mathrm{M}$ & $\mathrm{S} / \mathrm{C}$ \\
\hline Old clusters & $(2)$ & 1 & 10 & - & - & 0.10 \\
Int. clusters & $(12)$ & 6 & 19 & - & - & 0.32 \\
Young clusters & $(0)$ & 4 & 5 & - & - & 0.80 \\
LMC Bar SR & 27 & 13 & 22 & 1.30 & 0.81 & 0.59 \\
LMC Bar Miras & 11 & 2 & 7 & 0.82 & 0.64 & 0.29 \\
LMC Bar all & 37 & 15 & 27 & 1.14 & 0.73 & 0.56 \\
Galaxy SR & 2017 & 87 & 403 & 0.24 & 0.20 & 0.22 \\
Galaxy Mira & 1901 & 96 & 92 & 0.10 & 0.05 & 1.04 \\
Galaxy all & 3918 & 183 & 495 & 0.17 & 0.13 & 0.37 \\
Galaxy bright & 842 & 61 & 130 & 0.23 & 0.15 & 0.47 \\
\hline \hline
\end{tabular}

The number ratio of $\mathrm{S}$ to $\mathrm{C}$ stars among the $\mathrm{SR}$ variables in the Bar of the LMC is similar to that in the younger clusters. The number ratio of $\mathrm{S}$ to $\mathrm{C}$ stars is slightly higher in the LMC Bar field than in the galactic sample when we take Mira and semiregular variable stars together.

The ratio of the numbers of $\mathrm{S}$ to $\mathrm{C}$ stars is an indication of the relative time spent in the range of $\mathrm{C} / \mathrm{O}$ where s-processed material is being added to the convective envelope but the $\mathrm{C} / \mathrm{O}$ ratio has not yet been reversed. The spectral type at the $\mathrm{M}$ to $\mathrm{S}$ transition in the Magellanic Cloud star clusters depends on metal content (Bessell et al. 1983). The $\mathrm{M}$ to $\mathrm{S}$ and $\mathrm{S}$ to $\mathrm{C}$ transitions are both brighter in younger clusters and probably also in more metal-rich clusters (Lloyd Evans 1984). The relative scarcity of carbon stars with weak bands suggests a rapid transition from $\mathrm{O}$-rich to $\mathrm{C}$-rich, in line with theoretical expectations (Iben 
\& Renzini 1982), yet the abundance of MS and S stars suggests that there is a long interval during which the envelope is being enriched with heavy elements.

Theory indicates that $\mathrm{O}$-rich stars will be turned into carbon stars more easily at low metal content where there is less oxygen to counterbalance (Renzini \& Voli 1981; Iben 1981). Iben \& Truran (1978) suggested in addition that the $\mathrm{S}: \mathrm{C}$ ratio would be higher in a more metal-rich population. Iben \& Renzini (1982; see also Vanture, Wallerstein \& Suntzeff 2002) found that the O-rich to C-rich transition could be accomplished in only a few years in a star of very low mass, such as those in galactic globular clusters, leaving little room for an S-star phase unless the build-up of s-process elements in the envelope precedes that of carbon. This, however, is probably excluded by the observation that the $\mathrm{C} / \mathrm{O}$ ratio and ${ }^{12} \mathrm{C} /{ }^{13} \mathrm{C}$ both increase steadily along the sequence $\mathrm{M}-\mathrm{MS}-\mathrm{S}-\mathrm{C}$ among galactic field stars (Smith \& Lambert 1990). The cluster data suggest that the $\mathrm{S}$ star phase lasts relatively longer in younger clusters, in agreement with the prediction that the more massive envelopes require a greater number of dredgeup events to overturn the $\mathrm{C} / \mathrm{O}$ ratio. It may be that the lower metal content of the dominant population in the Bar of the LMC has been counterbalanced by younger age and correspondingly greater masses to yield an $\mathrm{S}: \mathrm{C}$ ratio which, by chance, is similar to that of the solar neighbourhood in the Galaxy.

\section{References}

Bessell, M.S., Wood, P.R., Lloyd Evans, T. 1983, MNRAS, 202, 59

Glass, I.S., Lloyd Evans, T. 1981, Nature, 291, 303

Glass, I.S., Liloyd Evans, T. 2003, MNRAS, 343, 67

Iben, I. 1981, ApJ, 246, 278

Iben, I., Renzini, A. 1982, ApJ, 259, L79

Iben, I., Truran, J.W. 1978, ApJ, 200, 980

Lloyd Evans, T. 1972, in The Magellanic Clouds, ed. A.B. Muller, (Dordrecht: Reidel), 74

Lloyd Evans, T. 1980, MNRAS, 193, 97

Lloyd Evans, T. 1983, MNRAS, 204, 985

Lloyd Evans, T. 1984, MNRAS, 208, 447

Lloyd Evans, T. 1992, in ASP Conf. Ser., Vol. 30, Variable Stars and Galaxies, ed. B. Warner (San Francisco: ASP), 169

Nishida, S., et al. 2000, MNRAS, 313, 136

Renzini, A., Voli, M. 1981, A\&A, 94, 175

Smith, V.V., Lambert, D.L. 1990, ApJS, 72, 387

Tanabe, T., et al. 1999, in IAU Symposium 191, Asymptotic Giant Stars, eds

T. LeBertre, A. Lebre, C. Waelkens, (San Francisco: ASP), 573

Vanture, A.D., Wallerstein, G., Suntzeff, N.B. 2002, ApJ, 569, 984 